\title{
Estimation of Fatigue Life of Long-Span Bridge by Considering Vehicle- Bridge Coupled Vibration
}

\author{
Ling Jiao*
}

School of Civil Engineering, Xuchang University, Xuchang Henan 461000, China

\begin{abstract}
To estimate the fatigue life of long-span steel bridge more accurately, this paper proposes a method for estimating the fatigue life of long-span bridges by considering vehicle-bridge coupled vibration. Firstly, the universal equation of vehicle-bridge vibration is derived based on triaxial standard multi-scale model of fatigue vehicle and bridge, and relevant program for vehicle-bridge coupled vibration is programmed. Secondly, a method for estimating the fatigue life of longspan steel bridge is proposed based on fracture mechanics theories, and the analysis process is expounded in details. Finally, the method for estimating fatigue life proposed in this paper is verified by taking a double pylon cable-stayed bridge with orthotropic steel bridge deck slab as an example.
\end{abstract}

Keywords: Fatigue life, Orthotropic steel bridge deck slab, Steel bridges, Vehicle-bridge coupled vibration.

\section{INTRODUCTION}

To cater to people's travel demand, there are more and more long-span bridges appearing everywhere in the world. However, with the increasing demand on long-span bridges, bridge designers have to face the intractable contradiction between bridge span and self-weight. Due to its large selfweight, traditional concrete bridge cannot meet the requirement of long-span bridges on small self-weight and large load-bearing of the girder. The occurrence of bridge with orthotropic steel bridge deck slab can solve this contradiction. Generally, orthotropic steel bridge deck slab is welded by mutually orthogonal bridge deck slabs, diaphragm plates and longitudinal ribbed stiffeners. The components bear the load synergistically under the vehicle load. However, the thin component plates are prone to causing stress concentration, thus causing fatigue crack [1]. If not emphasized, fatigue crack will often cause the collapse of in-use steel bridge; for instance, long-span steel bridges such as Japanese Fujigawa Bridge, US Hoan Bridge and Chinese Tsing Ma Bridge etc. all suffered fatigue crack damage and rupture to different extents $[2,3]$. Bridges bear circulating vehicle load during its normal operation, while the fatigue failure is a common failure form. Bridges, as an important traffic aisle, once damaged, will cause severe consequences. For long-span bridges, the impact effect of vehicles on the fatigue and sensitive parts of the bridges cannot be ignored. There are different calculation formulas for impact coefficients of bridges in the specifications in each country; however, the dynamic load of moving vehicles is all deemed as static load for calculation, with the coupling between moving vehicles and the bridge considered. In addition, for long-span

\footnotetext{
*Address correspondence to this author at the School of Civil Engineering, Xuchang University, Xuchang Henan 461000, China;

E-mail: Lingjiao1861@163.com
}

bridges, the stress histories at each fatigue detail part are different even in the same cross section [4].

To learn the stress characteristics of long-span steel bridges more accurately for guiding the anti-fatigue design of bridges, this paper plans to analyze vehicle-bridge coupled vibration of long-span bridge under multi-scale model, thus precisely considering the impact effect of vehicles' on the fatigue detail parts. Then, the researchers estimate the fatigue life of the bridge by means of fracture mechanics methods, and analyze the influences of various factors on the fatigue life of the bridge. Finally, they take a cable-stayed bridge with orthotropic steel bridge deck slab as an example to verify the proposed method for estimating the fatigue life of long-span bridges by considering the impact effect of vehicle-bridge coupled vibration.

\section{PRINCIPLE AND METHODS FOR ANALYZING VEHICLE-BRIDGE COUPLED VIBRATION}

\subsection{Standard Fatigue Vehicle}

There are corresponding provisions for standard fatigue vehicle models of highway bridges in the bridge design specifications of many foreign countries. However, there is no specific provision for this in China's specifications [5]. Given this background, triaxial fatigue vehicle model recommended in AASHTO specifications is adopted in this paper [6], where the headstock and trailer in the upper structure of a vehicle are deemed as two rigid bodies. The three axles of the vehicle is composed of mass block and spring-damping system; there are 11 degrees of freedom in this fatigue vehicle in total. Based on generalized virtual work principle, the differential equation of vehicle vibration system can be derived, which is as follows after being simplified to matrix form:

$$
\mathrm{M} \cdot \ddot{\mathrm{Z}}+\mathrm{C} \cdot \mathrm{Z}+\mathrm{K} \cdot \mathrm{Z}=\mathrm{G}+\mathrm{P}
$$


In the equation, $\mathrm{M}, \mathrm{C}$ and $\mathrm{K}$ are the mass, damping and stiffness matrix of the vehicle respectively; $Z, \dot{Z}$ and $\ddot{Z}$ are the generalized displacement, speed and accelerated speed vector; $\mathrm{G}$ is gravity column vector; $\mathrm{P}$ is the column vector of interaction between vehicle tyre and the bridge surface Fig. (1).

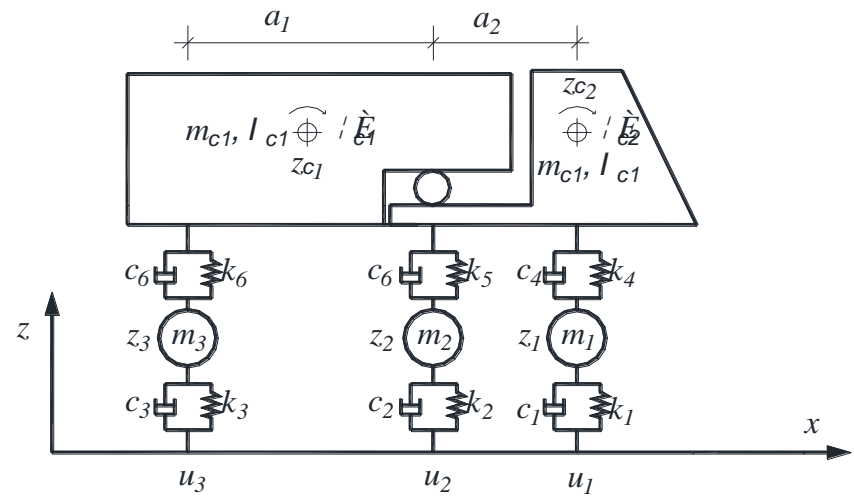

Fig. (1). Triaxial vehicle model.

\subsection{Vibration Equation and Multi-Scale Model of Bridges}

Generally, a bridge is the system and structure of multiple degrees of freedom and its vibration equation can be simplified as follows:

$$
\boldsymbol{M}_{\boldsymbol{b}} \ddot{Z}_{b}+\boldsymbol{C}_{\boldsymbol{b}} \dot{Z}_{b}+\boldsymbol{K}_{\boldsymbol{b}} Z_{b}=\boldsymbol{P}_{\boldsymbol{b}}
$$

In the equation, $\mathrm{Mb}, \mathrm{Cb}$ and $\mathrm{Kb}$ are the mass, damping and stiffness matrix of the bridge respectively; $\boldsymbol{P}_{\boldsymbol{b}}$ is vector of vehicle-bridge coupling applied on the bridge, $Z_{b}, \dot{Z}_{b}$ and $\ddot{Z}_{b}$ are the displacement, speed and accelerated speed vectors of the bridge respectively.

This paper programs the program for solving discretization and vibration equation of bridge structure model via Matlab and Ansys by means of Wilson- $\theta$.

As long-span bridges have long span and complicated structure, it will be time consuming to build a sophisticated model for the whole bridge, and the analysis process for its huge model data requires too much for computers, combined with low calculation efficiency, so that it's hard to realize it in reality. If equivalent simplification analysis is carried out on the whole of a bridge, the calculation efficiency will be significantly increased, but it will be difficult for the calculation precision to meet people's requirement. When analyzing long-span bridges, researchers only pay attention to the stress performance of some key parts instead of paying special attention to all the structure details. Therefore, sophisticated modeling can be carried out on the concerned parts, and large scale modeling can be carried out for other parts; the two are coupled by establishing constraint equations at the interfaces between models of different scales, i.e., mixed scale finite element model of long-span bridges are obtained, thus increasing both the calculation efficiency and the accuracy [7]. This paper plans to analyze the multi-scale model of long-span bridges by means of ANSYS finite element software.

\subsection{Simulation of bridge surface evenness}

Generally, the bridge surface evenness is deemed so as to meet the process of zero-mean stable Gauss random distribution [8]; its shape function $\mathrm{r}(\mathrm{x})$ can be simulated by the random process, and its expression is:

$r(x)=\sum_{i=1}^{n} \alpha_{i} \cos \left(2 \pi \beta_{i} x+\gamma_{i}\right)$

$\alpha_{i}^{2}=4 \cdot S_{r}\left(\beta_{i}\right) \cdot \Delta \beta$

$\beta_{i}=\beta_{1}+(i-1 / 2) \cdot \Delta \omega$

$\Delta \beta=\left(\beta_{u}-\beta_{1}\right)$

where $\alpha_{i}$ is the amplitude of cosine function, $\beta_{i}$ is the frequency of spatial-cut-off frequency in $\left[\beta_{1}, \beta_{u}\right]$ range, $\gamma_{i}$ is the evenly distributed starting phase angle in $[0,2 \pi]$ range, is the coordinate value of the bridge surface point along the longitudinal direction, $\mathrm{n}$ is the sum of the uneven points built. $S_{r}\left(\beta_{i}\right)$ is energy power spectral density function, it can be described as spatial frequency function of pavement unevenness in $\left[\beta_{1}, \beta_{u}\right][9]$, and its expression is:

$S_{r}\left(\beta_{i}\right)=\varepsilon \cdot \beta_{i}^{-\mu}, \beta_{i} \in\left(\beta_{1}, \beta_{n}\right)$

In the equation, $\varepsilon$ is relevant coefficient of each road unevenness; its values are taken according to Table $\mathbf{1}$.

The bridge surface unevenness is simulated by the aforesaid method, thus dividing the bridge surface unevenness into five grades of "excellent", "good", "general", "poor" and "very poor".

Table 1. Grades of bridge surface evenness.

\begin{tabular}{|c|c|c|}
\hline No. & Bridge Surface Grade & $\boldsymbol{\varepsilon} /\left(\mathbf{m}^{2} /(\mathbf{m} /\right.$ Cycle $\left.)\right)$ \\
\hline \hline 1 & Excellent & $\varepsilon<0.24 \times 10^{-6}$ \\
\hline 2 & Good & $0.24 \times 10^{-6}<\varepsilon<1 \times 10^{-6}$ \\
\hline 3 & General & $1 \times 10^{-6}<\varepsilon<4 \times 10^{-6}$ \\
\hline 4 & Poor & $4 \times 10^{-6}<\varepsilon<16 \times 10^{-6}$ \\
\hline 5 & Very poor & $16 \times 10^{-6}<\varepsilon$ \\
\hline
\end{tabular}

\subsection{Numerical Analysis Method for Vehicle-Bridge Coupled Vibration}

The vibrations equations of the bridge and the vehicle can be obtained based on virtual work principle and by virtue of finite element method. Based on the assumption that the vehicle tyres and the bridge surface are always in contact status, the two equations can be coupled by relation equations of displacement coordination and the interaction between the vehicle and the bridge, thus obtaining the final equation of vehicle-bridge coupled vibration. This paper programs corresponding program of vehicle-bridge coupled vibration based on Matlab and Ansys software in line with the aforesaid process.

\section{ESTIMATION OF BRIDGE FATIGUE LIFE BASED ON FRACTURE MECHANICS}

\subsection{Methods for Estimating the Fatigue Life of Bridges Based on Facture Mechanics}

The fatigue life of the structure often consists of emerging stage and propagation stage of the fatigue crack, where crack emerging stage includes the occurrence of tiny cracks and propagation in small range; propagation stage includes stable propagation of the crack and unstable fracture. As the fatigue 
life of engineering structure and components is mainly determined by the stable propagation stage of cracks, therefore, generally, the initial length of the fatigue cracks of the components are set according to test and empirical values when estimating fatigue life.

In fracture mechanics, $d a / d k$ usually deems the fatigue crack propagation rate as the function of the amplitude of stress intensity factor $\Delta K$ of the material; moreover, based on different crack propagation rates, the entire propagation process of fatigue crack can be divided into the first stage (crack emerging stage), the second stage (crack stable propagation stage) and the third stage (crack unstable fracture stage) $[10,11]$. To accurately study the fatigue crack propagation process, many scholars have put forward crack propagation rate model, among which the most classical one is crack propagation rate formula proposed by Paris and Erdogan based on linear elastic fracture mechanics; the formula is [12]:

$d a / d N=C \cdot(K)^{m}$

In the formula, a is crack depth, $\mathrm{N}$ is stress circle index, $\mathrm{C}$ and $m$ are material constants, as measured by tests, $\Delta K$ is the amplitude of stress intensity factor.

$\Delta K=K_{\max }-K_{\min }=Y \cdot \sigma \cdot \sqrt{\pi \cdot a}$

In the equation, $\mathrm{Y}$ is correction factor of stress intensity factor; $\sigma$ is the stress amplitude at the crack of the component.

Integral for Equation (8) can obtain the cyclic loading number that the structure can bear from initial crack depth $\mathrm{a} 0$ to failure crack depth af. The calculation formula for fatigue crack propagation life of steel bridge is:

$N=\int_{a_{0}}^{a_{\mathrm{f}}} \frac{1}{C \cdot K^{m}} d a$

where $\mathrm{a} 0$ is initial crack depth, af is final failure crack depth.

Solving Equation (10) can obtain the basic equation for estimating the calculation formula for fatigue crack propagation life of steel bridge based on fracture mechanics, as follow:

$$
\begin{aligned}
& N_{c}= \\
& \begin{cases}\frac{1}{C \cdot(f \cdot \Delta \sigma \cdot \sqrt{\pi})^{m}(0.5 m-1)} \cdot\left[\frac{1}{a_{0}^{(0.5 m-1)}}-\frac{1}{a_{f}^{(0.5 m-1)}}\right] & m \neq 2 \\
\frac{1}{C \cdot(f \cdot \Delta \sigma \cdot \sqrt{\pi})^{m}} \cdot \ln \left(\frac{a_{f}}{a_{0}}\right) & m=2\end{cases}
\end{aligned}
$$

When obtaining the cyclic loading number that a fatigue detail part of the bridge can bear during the entire crack propagation process, the annual traffic volume passing the bridge can be obtained through calculation; converting the actual annual traffic volume into standard traffic volume of fatigue vehicle shall obtain the fatigue life age limit of a certain part of the bridge, thus guiding the overhaul and maintenance time of the bridge.

\subsection{Influence of Vehicle-Bridge Coupled Vibration on the Fatigue Life}

In conventional estimation for the fatigue life of bridges, only the dynamic amplification of vehicle load on the bridges is considered by simple formula calculation, the coupling effect between moving vehicles and the bridge is not taken into consideration. Moreover, for long-span bridges with huge dimensions and complicated structure, the dynamic effects of each part are not uniform in actual status; therefore, a more precise method is needed to consider the influence of vehiclebridge coupled vibration on the fatigue life of steel bridges.

The influence of vehicle-bridge coupled vibration on the fatigue life of bridges are mainly reflected in the influence of fatigue vehicle on the stress intensity factors at the fatigue detail parts of the bridge; its influence factors mainly include selection of fatigue standard vehicle model, bridge surface evenness, vehicle speed, vehicle weight and traffic volume etc. The above-mentioned factors actually exist in the actual traffic on the bridge; only by taking into account these factors as far as possible can the fatigue life of the bridge be estimated more accurately. The influence of the above-mentioned influence factors affecting the fatigue life of bridges can be well considered by means of the program for analyzing vehicle-bridge coupled vibration of long-span bridge based on multi-scale model, and the stress histories at each fatigue detail part as well as the dynamic amplification effects by vehicle load can also be obtained more accurately.

In conclusion, the flow of the proposed method for estimating the fatigue life of long-span bridges by considering vehicle-bridge coupled vibration is as shown in Fig. (2).

\section{CASE ANALYSIS}

\subsection{Multi-Scale Finite Element Model of Double Pylon Cable-Stayed Bridge}

In this paper, analysis is carried out by taking a double pylon cable-stayed bridge as an example. This bridge is a river-cross highway cable-stayed bridge, with the span of 150 $m+300 m+150 \mathrm{~m}$ and bilateral eight lanes. The girder is orthotropic steel bridge deck slab, the main pylon is prestressed reinforced concrete structure, and the cable-stayed bridge is double inhaul cable semi-floating structure system. In this paper, a multi-scale finite element model of the whole bridge is built via Ansys, a large-scale universal finite element software, as shown in Fig. (3). The small-scale model segments are selected on the basis of existing detection results of the damaged parts of similar bridges as well as the analysis results of finite element model. In this paper, the mid-span of the main span is selected as the most adverse load-carrying part of the structure, i.e., the part with the minimum fatigue life. Based on the consideration for the safety of the entire structure, the fatigue life of this part determines the fatigue life of the whole bridge. Sophisticated models are built for smallscale models by Shell63 elements, the models for other girders and main pylon are built by large-scale Beam188 elements. The models of the two scales are connected at their interfaces by establishing constraint equations, thus obtaining the multiscale finite element model of this double pylon cable-stayed bridge. Based on this model, different fatigue detail parts can be selected for sophisticated anti-fatigue analysis on the structure. 


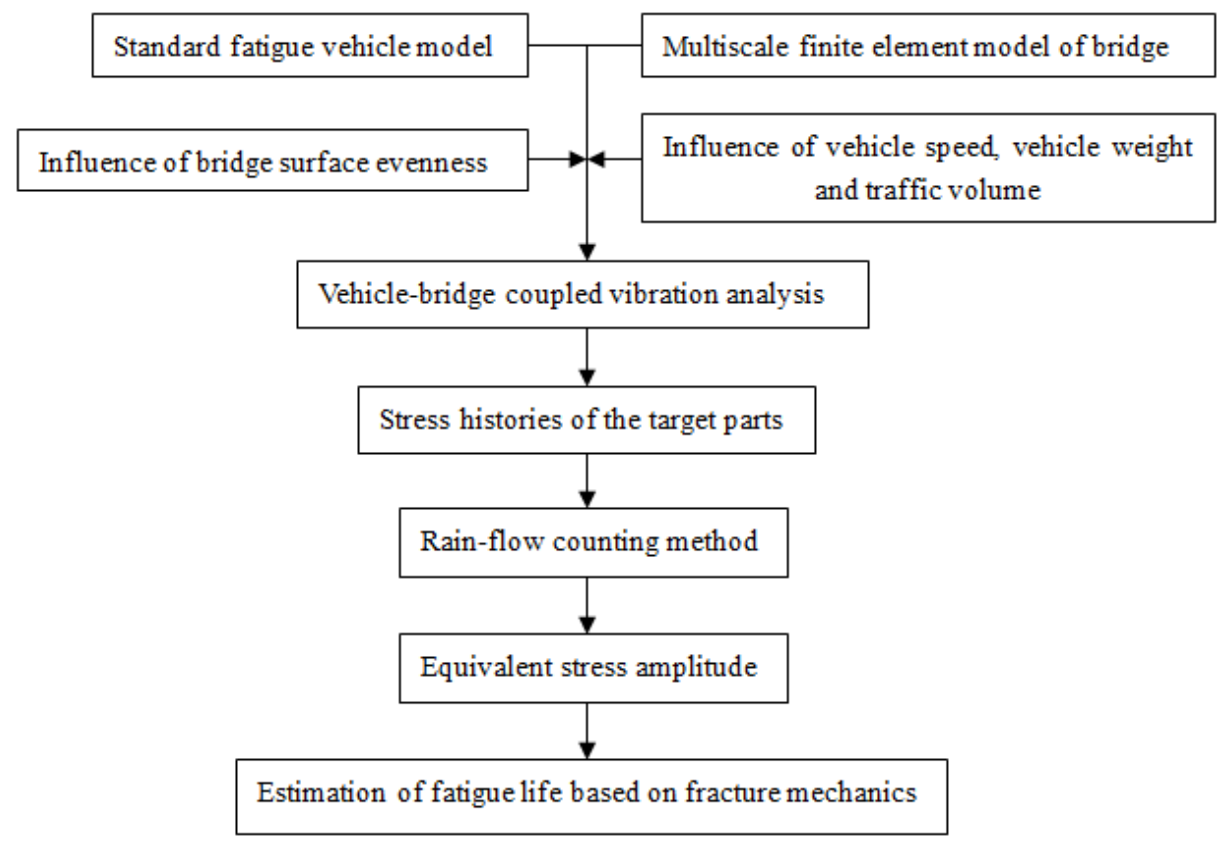

Fig. (2). Flow chart of fatigue life estimation.

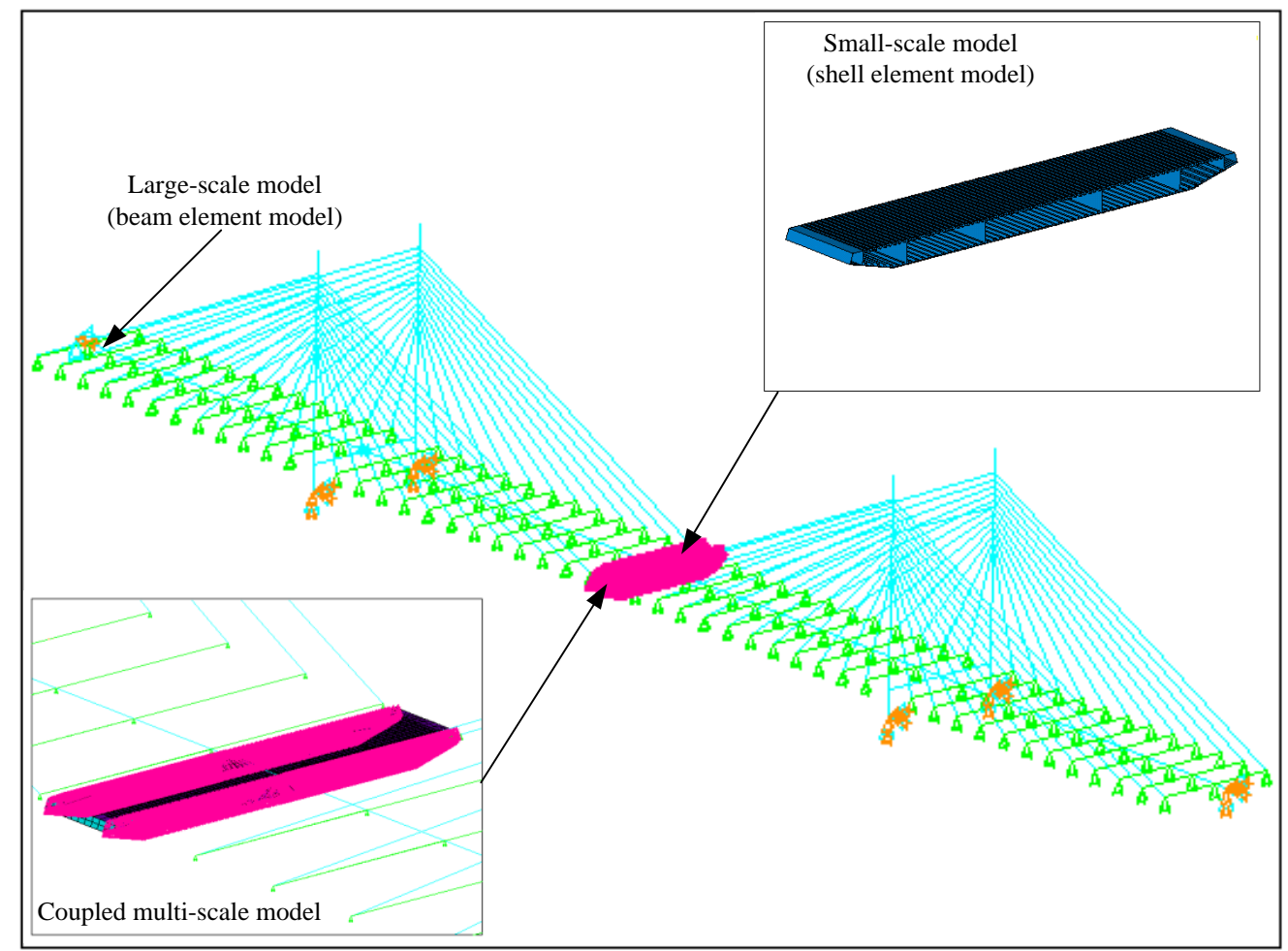

Fig. (3). Multi-scale finite element model of double pylon cable-stayed bridge.

\subsection{Estimation for the Fatigue Life of Cable-stayed Bridge}

Based on the method for estimating the fatigue life of longspan bridges by considering vehicle-bridge coupled vibration proposed in this paper, fatigue analysis can be carried out by selecting different fatigue details from local small-scale models, thus obtaining the fatigue life of different fatigue detail part in the same cross section to guide anti-fatigue design of the bridges. In this paper, the two kinds of common cracks in actual orthotropic steel bridge deck slab are selected to carry out analysis, i.e., crack $\mathrm{A}$ at the welding part between $\mathrm{U}$ rib and crossbeam and crack $\mathrm{B}$ at the welding part between top plate and U rib, as shown in Fig. (4).

The stress histories of each fatigue detail part can be obtained through vehicle-bridge coupled vibration analysis on multi-scale bridge model by fatigue vehicle, and the stress amplitudes of corresponding parts can be obtained by rainflow counting method, and its fatigue life can be estimated based on fracture mechanics methods. The fatigue cracks at A 

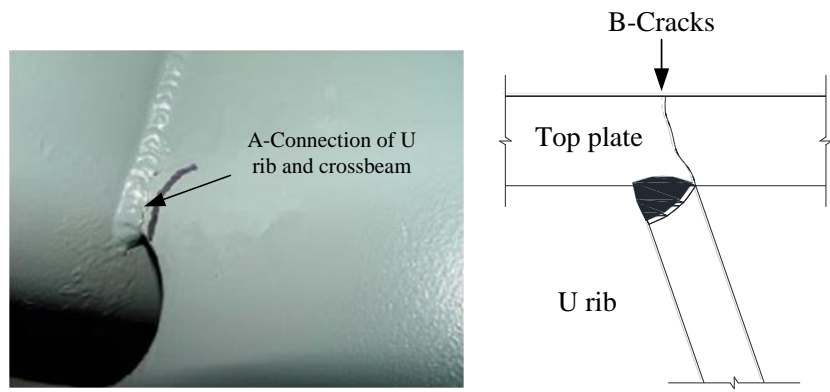

Fig. (4). Details of the fatigue crack in orthotropic steel bridge deck slab.

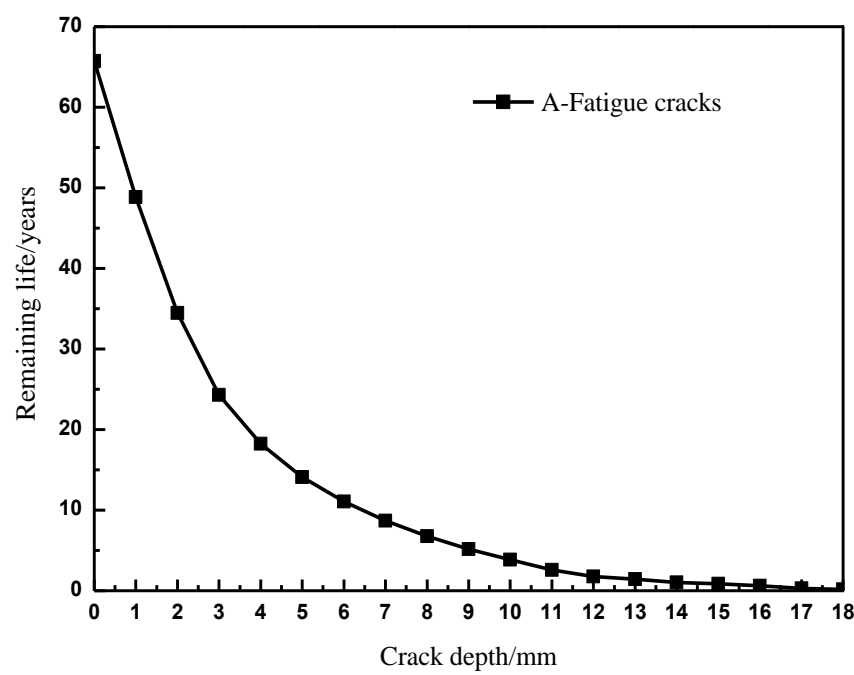

Fig. (5). Analysis diagram for the fatigue crack propagation life at A of double pylon cable-stayed bridge.

and $B$ are analyzed by the proposed methods; the fatigue crack propagation life at $\mathrm{A}$ and $\mathrm{B}$ are as shown in Figs. $(\mathbf{5}, \mathbf{6})$ respectively. According to the slopes of the fatigue life curves in Figs. $(\mathbf{5}, \mathbf{6})$, the fatigue detail sees slow propagation rate at early crack propagation stage and fast propagation rate during middle and late stage. The fatigue life of the fatigue detail at A shortens to half as that at B, which indicates that the fatigue life of different fatigue detail parts on the same cross section of this steel girder also differs. This also reflects the different stress concentrations at each fatigue detail part. The fatigue life difference between $\mathrm{A}$ and $\mathrm{B}$ also properly reflects the advantages of the method for estimating the fatigue life of long-span bridge by considering vehicle-bridge coupled vibration based on multi-scale model, i.e., sophisticated fatigue analysis can be carried out on each fatigue detail part.

\subsection{Influence of bridge surface evenness on the fatigue life of the steel bridge}

To research the influence of bridge surface evenness on the fatigue life of the steel bridge, in this paper, the bridge surface evenness is divided into five grades of "excellent", "good", "general", "poor" and "very poor" in vehicle-bridge coupled vibration program. Based on the proposed analysis process, the fatigue life at $\mathrm{A}$ and $\mathrm{B}$ under five kinds of bridge surface evenness are analyzed respectively; the analysis results are as shown in Figs. $(\mathbf{7}, \mathbf{8})$ respectively. According to

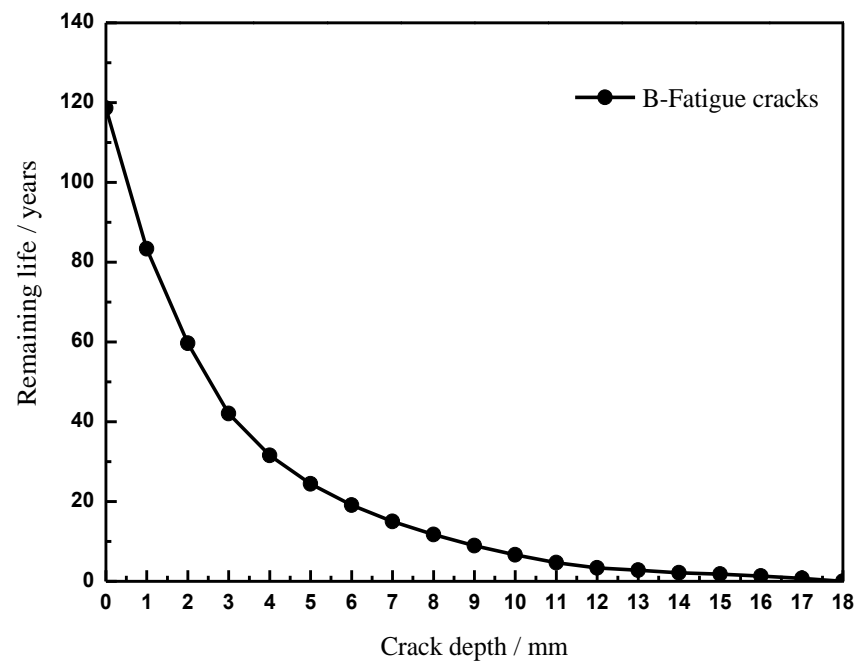

Fig. (6). Analysis diagram for the fatigue crack propagation life at B of double pylon cable-stayed bridge.

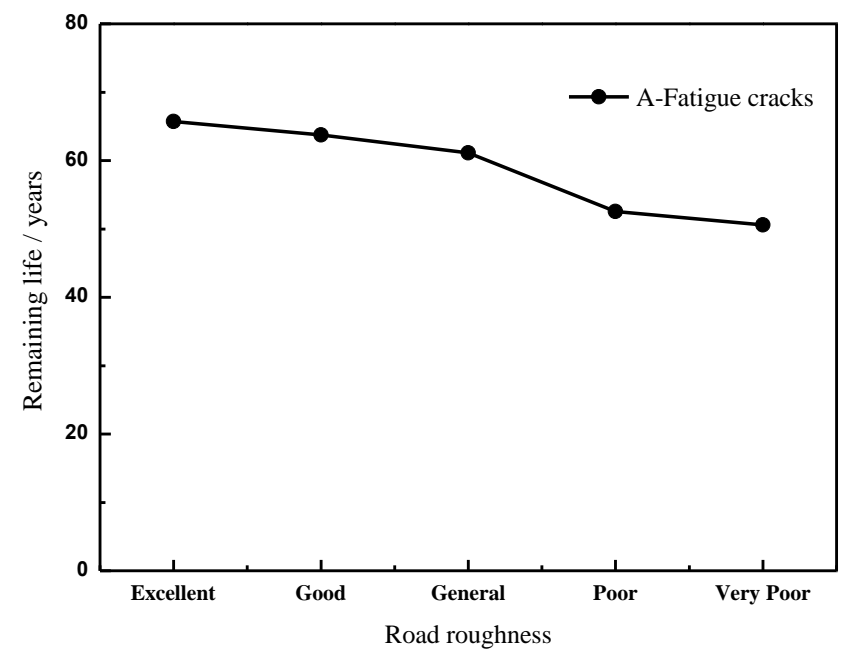

Fig. (7). Influence of bridge surface evenness on the fatigue life at A.

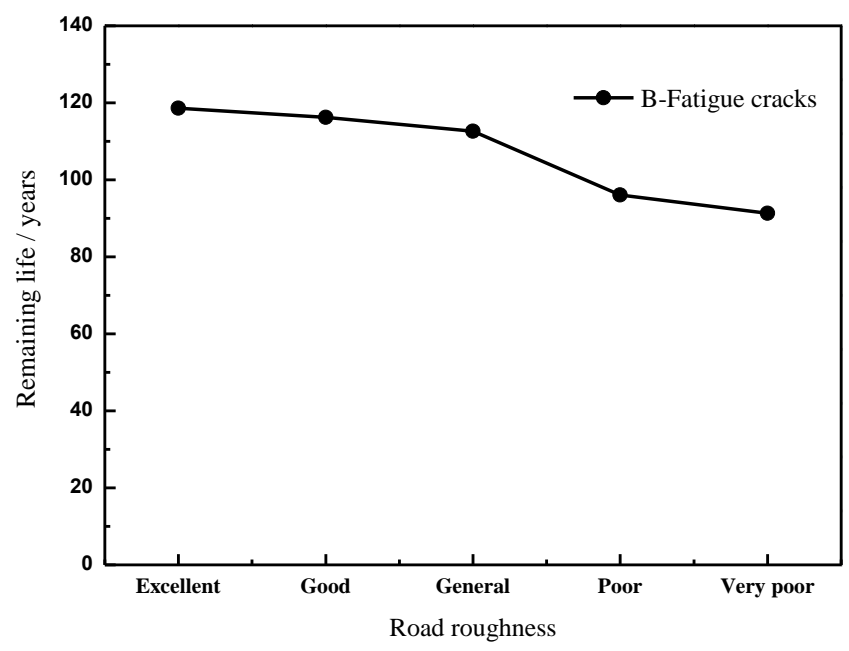

Fig. (8). Influence of bridge surface evenness on the fatigue life at B.

Figs. $(7,8)$, the fatigue life at A and B decreases as the bridge surface evenness deteriorates. The fatigue life reduces to a small extent when the bridge surface evenness are "excellent", "good" and "general", i.e., the fatigue life at these moments is 
not sensitive to the three road conditions. However, when the bridge surface evenness deteriorates to "poor" and "very poor", the fatigue life will reduce significantly.

\section{CONCLUSION}

(1) The amplification of vehicle-bridge coupling on the stress at fatigue detail part cannot be ignored when carrying out fatigue analysis on long-span bridges.

(2) The proposed method for estimating the fatigue life of long-span bridge by considering vehicle-bridge coupled vibration can consider vehicle-bridge interaction, vehicle model, bridge surface evenness and vehicle speed. Moreover, based on the multi-scale finite element model, it can select the stress history at any fatigue detail part to conduct analysis, thus obtaining more accurate results when carrying out fatigue analysis on the bridge.

(3) According to the fatigue analysis on the double pylon cable-stayed bridge in this paper, the fatigue life at each fatigue detail part are different; when the bridge surface evenness deteriorates to "poor" and "very poor", the fatigue life will reduce significantly.

\section{CONFLICT OF INTEREST}

The author confirms that this article content has no conflict of interest.

\section{ACKNOWLEDGEMENTS}

This project is supported by Fundamental Research Funds for the Central Universities (No. CHD2011JC155)

\section{REFERENCES}

[1] X. Y. Tao, X. G. Liu, and Y. L. Zhang, "Study on the stress characteristics of orthotropic steel deck", Steel Construction, vol. 25, no. 7, pp. 12-14, 2010.

[2] S. B. Lee, "Fatigue failure of welded vertical members of a steel truss bridge," Engineering Failure Analysis, vol. 3, no. 2, pp. 103-108, 1996.

[3] K. Y. Wong, "Structural identification of tsing ma bridge," Transaction of the Hong Kong Institute of Engineers, vol. 10, no.1, pp. 38-47, 2003.

[4] Z. X. Li, T. Q. Zhou, T. H. T. Chan, and Y. Yu, "Multi-scale numerical analysis on dynamic response and local damage in longspan bridge," Engineering Structures, vol. 29, no.7, pp. 1507-1524, 2007.

[5] L. W. Tong, Z. Y. Shen, and Z. Y. Chen, "Fatigue load spectrum for urban road bridges," China Civil Engineering Journal, vol. 30, no.5, pp. 20-27, 1997.

[6] AASHTO, AASHTO LRFD bridge design specification, USA: AASHTO, 1994.

[7] K. Maekawa, T. Ishida, and T. Kishi, "Multi-scale modeling of concrete performance integrated material and structural mechanics," Journal of Advanced Concrete Technology, pp. 91-126, 2003.

[8] F. T. K. Au, Y. S. Cheng, and Y. K Cheung, "Effects of random road surface roughness and long-term deflection of prestressed concrete girder and cable-stayed bridges on impact due to moving vehicles," Computers and Structures, vol. 79, pp. 853-872, 2001.

[9] H. Honda, Y. Kajikawa, and T. Kobori, "Spectra of road surface roughness on bridges," Journal of Structural Division ASCE, vol. 108, pp. 1956-1966, 1982.

[10] J. Cheng, and S. S. Zhao, Fracture Mechanics, Science Press, Beijing, 2006, pp. 147-154.

[11] A. G. Zhang, J. C. Zhu, and M. C. Chen, Fatigue, Fracture and Damage, Southwest Jiaotong University Press, Chengdu, 2006.

[12] P. C. Paris, M. P. Gomez, W. P. Anderson, "A rational analytic theory of fatigue," Trend in Engineering, vol. 13, pp. 9-14, 1961. 